\title{
Pandemic considerations in pediatric critical care: what can we learn from COVID-19?
}

\author{
Tiantian Xiao $^{1 \#}$, Ye Cheng ${ }^{2 \#}$, Gouping Lu ${ }^{2}$, Wenhao Zhou ${ }^{1}$ \\ ${ }^{1}$ Department of Neonatology, Children's Hospital of Fudan University, Shanghai, China; ${ }^{2}$ Pediatric Intensive Care Unit, Children's Hospital of \\ Fudan University, Shanghai, China \\ "These authors contributed equally to this work. \\ Correspondence to: Gouping Lu. Pediatric Intensive Care Unit, Children's Hospital of Fudan University, 399 Wanyuan Road, Shanghai, China. \\ Email: lgp@fudan.edu.cn; Wenhao Zhou. Department of Neonatology, Children's Hospital of Fudan University, Shanghai, China. Email: \\ zhouwenhao@fudan.edu.cn.
}

Submitted Apr 27, 2020. Accepted for publication Nov 06, 2020.

doi: $10.21037 /$ tp-20-129

View this article at: http://dx.doi.org/10.21037/tp-20-129

Since December 2019, coronavirus disease 2019 (COVID-19), caused by severe acute respiratory syndrome coronavirus 2 (SARS-CoV-2), has been spreading around the world. On March 11th 2020, the World Health Organization (WHO) declared COVID-19 a pandemic. The SARS-CoV-2 virus is highly contagious among the general population.

So far, a large number of reports have analyzed critical COVID-19 cases in adults; however, the data on critical pediatric patients with the disease are limited. Consequently, many questions on COVID-19 in children still need to be answered. Why are children less likely to be affected by the disease than adults? Can neonates be infected via vertical transmission? Can children transmit SARS-CoV-2 to other people? What are the best treatment strategies for children with critical COVID-19? What are the neurological outcomes of children with COVID-19? Why do some children present with hyperinflammatory syndrome after SARS-CoV-2 infection?

With the number of infections across the world increasing, pediatricians are faced with finding solutions to these challenges.

\section{Epidemiological and clinical features of children with COVID-19}

Data from a Chinese cohort suggested that children of all ages are susceptible to SARS-CoV-2, with $2.2 \%$ of individuals with COVID-19 being under the age of 19 years old (1). In the United States, children account for only 2\% of COVID-19 cases (2); in Italy and Spain, this proportion has been reported to be $1.2 \%$ (3) and $0.8 \%$ (4), respectively. Chinese studies have suggested that in Wuhan, where COVID-19 was first reported, the majority of pediatric cases got infected at home during the early stages of the outbreak $(5,6)$.

More than $90 \%$ of children with COVID-19 are asymptomatic, mild, or moderate cases. According to Dong et al.'s study, most children with COVID-19 in China have shown favorable outcomes, with a median duration of hospitalization of 10 days (range, 8-14.3 days) (7). Moreover, a study from New York City reported the median length of hospital stay for most children to be 3 days (range, 1-30 days) (8). Meanwhile, the incubation time and the virus shedding time in children range from 1-14 days (mean, 3-7 days), and 3-4 weeks, respectively (9).

Common symptoms of lab-confirmed pediatric COVID-19 cases include flu-like symptoms, such as fever and cough, which are clinically indistinguishable from other infections (10). However, in early April 2020, cases of children with multisystem inflammatory syndrome (MIS-C) were reported to be increasing in Europe (11-13). One study suggested that SARS-CoV-2 infection might trigger a severe form of Kawasaki disease (KD), with pediatric cases presenting with classical or incomplete types of $\mathrm{KD}$ (13). In the same study, $50 \%(5 / 10)$ of infected children presented with KD shock syndrome (KDSS). Although this Kawasakilike disease can result in infected children becoming 
critically unwell, no mortalities have been reported $(11,13)$. In China, no cases of Kawasaki-like disease have been recorded to date.

A recent study described the rate of COVID-19 coinfection with other respiratory pathogens, including influenza, parainfluenza, rhinovirus/enterovirus, respiratory syncytial virus, metapneumovirus, other coronavirus, and mycoplasma pneumonia, to be $20.7 \%$ (24/116) (14). In contrast, the rate of viral co-infection in a European cohort was only $5 \%(29 / 582)$, and an early study reported that $4.0 \%$ (4/99) of COVID-19 patients were co-infected with bacteria or fungus $(15,16)$.

Zhang et al.'s study, which described the immunity features of six infected children, suggested that a rapid protective humoral immunity could be detected following SARS-CoV-2 infection (17). No difference was observed in CD4 or CD8 cell count between infected and uninfected children. Currently, the pathophysiology of multisystem inflammatory syndrome after SARS-CoV-2 infection is unclear.

Infected children have rarely been observed to have the abnormal laboratory results commonly seen in adult COVID-19 patients, which include leukopenia, lymphocytopenia, elevated transaminase, lactate dehydrogenase (LDH), $\alpha$-hydroxybutyrate dehydrogenase $(\alpha-\mathrm{HBDH})$, interleukin-6 (IL-6), and lung radiological findings $(18,19)$. However, no studies have demonstrated a consistent pattern in the laboratory results of children with COVID-19. Radiological findings of infected children are either normal or show increased lung markings (5). Abnormal findings on chest computed tomography (CT) scans include ground-glass opacity, local or bilateral patchy shadowing, and interstitial abnormalities (10). While chest CT has high sensitivity, its specificity is low, which makes distinguishing COVID-19 from other diseases challenging (10). Moreover, the high radiation exposure with chest CT limits its application for neonates and infants. Some studies have been initiated to investigate the findings of lung ultrasound in children with COVID-19. One study found that the lung ultrasonic findings of COVID-19 pneumonia in neonates with COVID-19 included abnormal pleura line, confluent B-lines, alveolar interstitial syndrome (AIS), or local consolidation (20).

According to Shekerdemian et al.'s study, up to $83 \%$ of children with COVID-19 in North American intensive care units (ICUs) have a comorbidity, such as obesity, immune suppression/malignancy, or congenital heart disease (21). A European cohort study described that male children under the age of 1 month with comorbidities and symptoms of lower respiratory tract infection had a higher risk of ICU admission (15). Another study indicated that higher C-reactive protein and procalcitonin levels at admission, as well as elevated peak IL-6, ferritin, and D-dimer levels during hospitalization, were associated with severe COVID-19 (21). Regarding Kawasaki-like disease after SARS-CoV-2 infection, age over 5 years and ferritin higher than $1,400 \mu \mathrm{g} / \mathrm{L}$ were associated with poor prognosis (22).

\section{Diagnosis}

The initial identification of COVID-19 in children could be complicated by the atypical clinical findings and possibly large number of asymptomatic children with the disease. An earlier study described as many as $12.8 \%$ (94/731) of children with confirmed COVID-19 to be asymptomatic (7). Another recent study reported that $13.7 \%$ of pregnant women who tested positive for SARS-Cov-2 on admission had no symptoms (23). Therefore, for children with flulike symptoms or a possible of history of exposure to COVID-19, pediatricians should have high suspicion and a low threshold for diagnostic testing.

The seventh edition of the New Coronavirus Pneumonia Prevention and Control Protocol for COVID-19 recommends reverse transcriptase-polymerase chain reaction (RT-PCR) assays and serological testing (IgM/IgG) for patient diagnosis. RT-PCR and bacterial culture of upper (nasopharyngeal) or lower (induced sputum, endotracheal aspirates, bronchoalveolar lavage) respiratory tract samples, rectal swabs, and stool and blood samples can be used to detect SARS-CoV-2 and other causative pathogens (24). A study on the pattern of viral excretion from the respiratory and gastrointestinal tracts in 10 confirmed pediatric COVID-19 cases demonstrated that the virus shedding time in feces was longer than that in respiratory samples (25). Consistent with this result, the rectal swabs from two neonates were found to test positive for SARS-CoV-2 after nasopharyngeal swabs returned negative results $(18,26)$. Therefore, some authors have suggested rectal swab-testing to be the better marker for validating the effectiveness of treatment (25). In additional, as stated above, the radiological findings of children with COVID-19 are atypical and have differed from study to study (27).

One study reported that IgM and IgG antibody titers for SARS-CoV-2 have been detected in the serum of neonates with COVID-19 at birth (28). However, no series of antibody tests has been performed; thus, false positive 


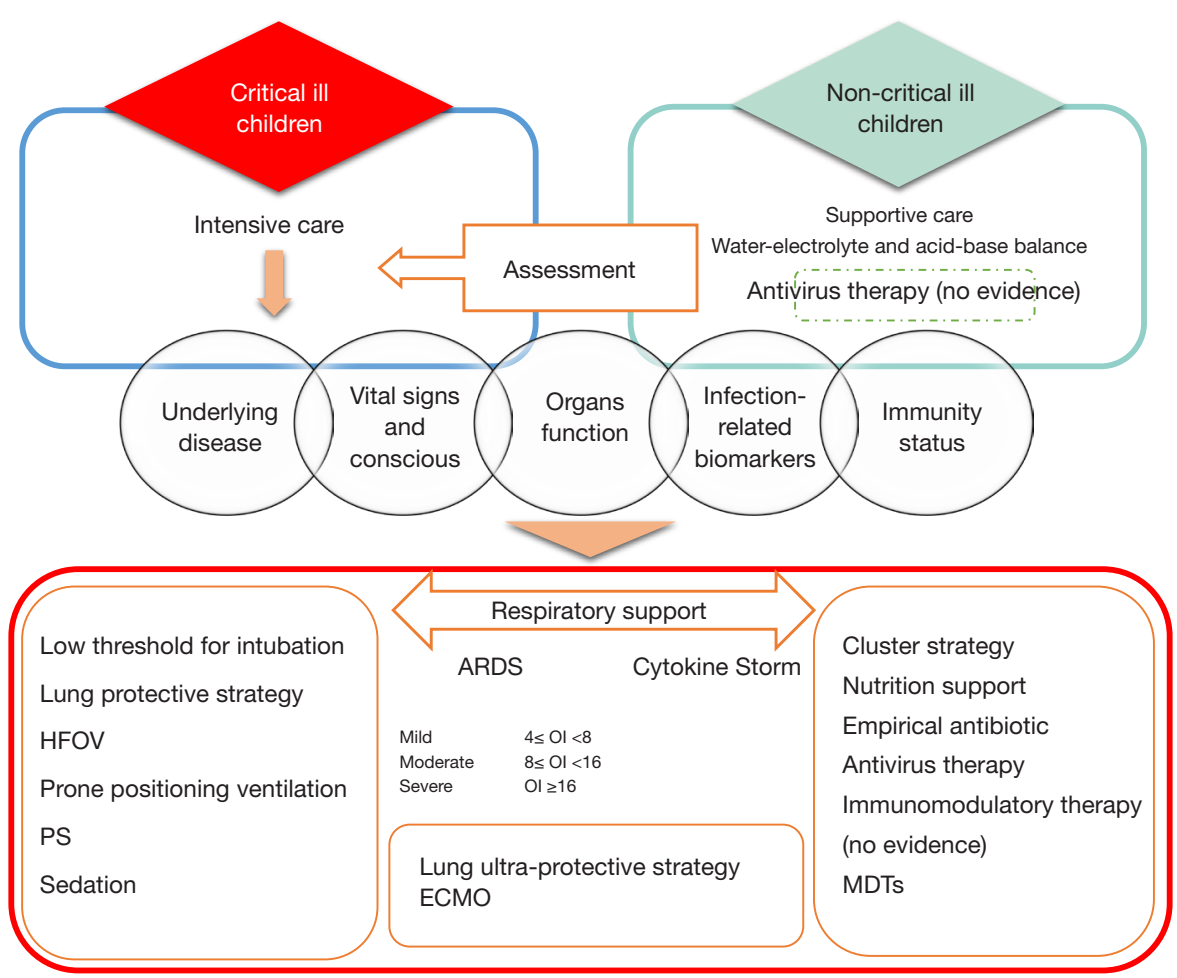

Figure 1 Clinical management of children with coronavirus disease 2019 (30-33). HFOV, high-frequency oscillatory ventilation; PS, pulmonary surfactant; iNO, inhaled nitric oxide; ARDS, acute respiratory distress syndrome; MDTs, multi-disciplinary teams; ECMO, extracorporeal membrane oxygenation.

results cannot be ruled out. So far, investigations of the immune response features of children with COVID-19 have been based on the limited data, and whether the dynamic changes in the virus load and antibody titers are closely related to the clinical course of the disease is unknown (29).

\section{Effective intervention for children with critical CoVID-19 (Figure 1)}

Early and appropriate medical support is crucial for children with COVID-19. Notably, the clinical management of children with COVID-19 is based on the findings of observational studies rather than clinical trials. Also, most of the randomized clinical trials carried out so far did not recruit participants under the age of 18 years, and limited therapeutic data have been published. Therefore, at this stage, the management recommendations should be based on children's physiological characteristics and the pathological features of COVID-19, taking some leads from clinical research involving adults with COVID-19.
In China, a large cohort study showed that $11 \%$ $(8,255 / 72,314)$ of COVID-19 cases in adults were critical (1), while only $5.8 \%(125 / 2,143)$ of pediatric patients with COVID-19 presented with severe illness (7). A European multicenter study showed that $8 \%(48 / 582)$ of children with COVID-19 required admission to an ICU (15). During the epidemic period in Wuhan, three severe pediatric cases with lab-confirmed COVID-19 were reported (7). One child died of sepsis and gastrointestinal dysfunction. Of the two surviving children, one child had gastrointestinal symptoms from the onset and went on to develop acute respiratory distress syndrome (ARDS), shock, and acute kidney injury (AKI), and the other, who had acute leukemia, progressed to ARDS. All three children received mechanical ventilation in the prone position for 6 to 12 hours each day, as well as continuous renal replacement therapy (CRRT), inotropes and vasoactive drugs, methylprednisolone, and antiviral therapy (interferon $\alpha-1 b$ inhalation and oseltamivir). However, none of the children required extracorporeal membrane oxygenation (ECMO) support. All three children had come into contact with adults who tested positive for COVID-19. 
Two studies reported that children with multisystem inflammatory syndrome or KDSS showed a good response to adjunctive steroid treatment, intravenous immunoglobulin, aspirin, and inotropic treatment $(11,13)$.

As stated by the Chinese Pediatric Novel Coronavirus Study Team and the Chinese perinatal-neonatal SARSCoV-2 Committee, children with COVID-19 should be managed according to the severity of their disease (30). Most children improve with supportive treatment, including oxygen therapy and maintenance of the water, electrolyte, and acid-base balance. However, children with underlying pulmonary diseases, immune deficiency, or immunocompromised status should be monitored closely (30). If the disease does not follow the clinical course expected, children with comorbidities should be further investigated. For children with critical COVID-19, respiratory support is essential. As the pathological manifestations of SARS-CoV-2 infection in the lung are the same as those of ARDS, with pulmonary edema, hyaline membrane formation, bilateral diffuse alveolar damage with cellular fibromucoid, and thick mucinous secretions in the trachea (31), the respiratory management strategy should follow the pediatric ARDS evidence-based guidelines (32). For children with COVID-19, a lung protective ventilation strategy including low tidal volume, optimized positive endexpiratory pressure (PEEP) and target oxygenation, limited plateau pressure, and permissive hypercapnia should be adopted. Multiple organs can be affected in critical pediatric COVID-19 cases (31), therefore, multiple organ support should be considered.

It should be kept in mind that COVID-19 patients can be co-infected with other pathogens. Therefore, empirical antibiotic and antiviral therapy should be administered based on the results of microbiology testing and clinical response. The roles of corticosteroids and immunoglobulin therapy remain uncertain. Although the current evidence suggests that COVID-19 is associated with a cytokine storm, no randomized controlled trial has demonstrated the efficacy and safety of steroids or immunoglobulin therapy in the treatment of children with COVID-19. Many randomized controlled trials have been launched to identify effective antiviral treatments. However, no antiviral therapies have been proven effective to date (33).

\section{Protecting children and healthcare workers in the ICU}

Because COVID-19 is highly contagious and aerosol- generating procedures (e.g., tracheal intubation, tracheostomy, cardiopulmonary resuscitation, bag-mask ventilation, and bronchoscopy) are frequently used in the ICU, personal protective equipment (PPE), closed suctioning systems, and viral filters should be used to protect healthcare workers and to prevent the spread of nosocomial infection. Healthcare workers should also be given COVID-19 prevention training.

\section{Early quarantine and emotional support}

Effective measures for screening patients, including the quarantining of suspected and confirmed patients, should be put in place. However, isolation and loneliness are increasing as a result of the pandemic. Mother-infant separation and the quarantining of infected children may cause anxiety to children and their families; thus, psychological support should be offered. Social workers can help patients, families, and healthcare workers. Mobile visiting services can also be used in neonatal and pediatric ICUs, as studies have demonstrated that telemental health services are as effective as in-person services over the short term (34).

\section{Conclusions}

The clinical features of COVID-19 are nonspecific in children, and few pediatric cases progress to severe disease. Current management strategies are based on limited evidence. Nevertheless, a low threshold for diagnostic testing, early diagnosis and quarantine, close monitoring of potentially sick children, early and appropriate medical treatment, a clustering strategy, and multi-disciplinary team (MDT) involvement are important for the management of children with COVID-19. Most of all, collaboration between patients, families, and healthcare workers will give critically ill children the best chances of survival and having a good prognosis during this pandemic.

\section{Acknowledgments}

Funding: None.

\section{Footnote}

Provenance and Peer Review: This article was commissioned by the Guest Editors (Jan Hau Lee, Vijay Srinivasan, and Debbie Long) for the series "Pediatric Critical Care" 
published in Translational Pediatrics. The article has undergone external peer review.

Peer Review File: Available at http://dx.doi.org/10.21037/tp20-129

Conflicts of Interest: All authors have completed the ICMJE uniform disclosure form (available at http://dx.doi. org/10.21037/tp-20-129). The series "Pediatric Critical Care" was commissioned by the editorial office without any funding or sponsorship. The authors have no other conflicts of interest to declare.

Ethical Statement: The authors are accountable for all aspects of the work in ensuring that questions related to the accuracy or integrity of any part of the work are appropriately investigated and resolved.

Open Access Statement: This is an Open Access article distributed in accordance with the Creative Commons Attribution-NonCommercial-NoDerivs 4.0 International License (CC BY-NC-ND 4.0), which permits the noncommercial replication and distribution of the article with the strict proviso that no changes or edits are made and the original work is properly cited (including links to both the formal publication through the relevant DOI and the license). See: https://creativecommons.org/licenses/by-nc-nd/4.0/.

\section{References}

1. Wu Z, McGoogan JM. Characteristics of and Important Lessons From the Coronavirus Disease 2019 (COVID-19) Outbreak in China: Summary of a Report of 72314 Cases From the Chinese Center for Disease Control and Prevention. JAMA 2020;323:1239-42.

2. CDC COVID-19 Response Team. Coronavirus Disease 2019 in Children - United States, February 12-April 2, 2020. MMWR Morb Mortal Wkly Rep 2020;69:422-6.

3. Livingston E, Bucher K. Coronavirus Disease 2019 (COVID-19) in Italy. JAMA 2020;323:1335.

4. Tagarro A, Epalza C, Santos M, et al. Screening and Severity of Coronavirus Disease 2019 (COVID-19) in Children in Madrid, Spain. JAMA Pediatr 2020. [Epub ahead of print].

5. Wei M, Yuan J, Liu Y, et al. Novel Coronavirus Infection in Hospitalized Infants Under 1 Year of Age in China. JAMA 2020;323:1313-4.

6. Liu W, Zhang Q, Chen J, et al. Detection of Covid-19 in
Children in Early January 2020 in Wuhan, China. N Engl J Med 2020;382:1370-1.

7. Dong Y, Mo X, Hu Y, et al. Epidemiological Characteristics of 2143 Pediatric Patients With 2019 Coronavirus Disease in China. Pediatrics 2020. doi: 10.1542/peds.2020-0702

8. Zachariah P, Johnson CL, Halabi KC, et al. Epidemiology, Clinical Features, and Disease Severity in Patients With Coronavirus Disease 2019 (COVID-19) in a Children's Hospital in New York City, New York. JAMA Pediatr 2020;174:e202430.

9. The Society of Pediatrics, Chinese Medical Association, the Editorial Board. Chinese Journal of Pediatrics Recommendations for the diagnosis, prevention and control of the 2019 novel coronavirus infection in children (first interim edition). Chin J Pediatr 2020. [Epub ahead of print].

10. Lu X, Zhang L, Du H, et al. SARS-CoV-2 Infection in Children. New Engl J Med 2020;382:1663-5.

11. Riphagen S, Gomez X, Gonzalez-Martinez C, et al. Hyperinflammatory shock in children during COVID-19 pandemic. Lancet 2020;395:1607-8.

12. Jones VG, Mills M, Suarez D, et al. COVID-19 and Kawasaki Disease: Novel Virus and Novel Case. Hosp Pediatr 2020;10:537-40.

13. Verdoni L, Mazza A, Gervasoni A, et al. An outbreak of severe Kawasaki-like disease at the Italian epicentre of the SARS-CoV-2 epidemic: an observational cohort study. Lancet 2020;395:1771-8.

14. Kim D, Quinn J, Pinsky B, et al. Rates of Co-infection Between SARS-CoV-2 and Other Respiratory Pathogens. JAMA 2020;323:2085-6.

15. Götzinger F, Santiago-García B, Noguera-Julián A, et al. COVID-19 in children and adolescents in Europe: a multinational, multicentre cohort study. Lancet Child Adolesc Health 2020;4:653-61.

16. Chen NS, Zhou M, Dong X, et al. Epidemiological and clinical characteristics of 99 cases of 2019 novel coronavirus pneumonia in Wuhan, China: a descriptive study. Lancet 2020;395:507-13.

17. Zhang $\mathrm{Y}, \mathrm{Xu}$ J, Jia R, et al. Protective humoral immunity in SARS-CoV-2 infected pediatric patients. Cell Mol Immunol 2020;17:768-70.

18. Holshue ML, DeBolt C, Lindquist S, et al. First Case of 2019 Novel Coronavirus in the United States. New Engl J Med 2020;382:929-36.

19. Henry BM, Lippi G, Plebani M. Laboratory abnormalities in children with novel coronavirus disease 2019. Clin 
Chem Lab Med 2020;58:1135-8.

20. Feng X, Tao X, Zeng L, et al. Application of pulmonary ultrasound in the diagnosis of COVID-19 pneumonia in neonates. Chin J Pediatr 2020. [Epub ahead of print].

21. Shekerdemian LS, Mahmood NR, Wolfe KK, et al. Characteristics and Outcomes of Children With Coronavirus Disease 2019 (COVID-19) Infection Admitted to US and Canadian Pediatric Intensive Care Units. JAMA Pediatr 2020;174:868-73.

22. Pouletty M, Borocco C, Ouldali N, et al. Paediatric multisystem inflammatory syndrome temporally associated with SARS-CoV-2 mimicking Kawasaki disease (KawaCOVID-19): a multicentre cohort. Ann Rheum Dis 2020;79:999-1006.

23. Goffman DS, Karin F, Mary DA. Universal Screening for SARS-CoV-2 in Women Admitted for Delivery. Available online: https://dx.doi.org/10.1056/NEJMc2009316

24. National Health Commission of the People's Republic of China. New Coronavirus Pneumonia Prevention and Control Protocol, 7th ed. Available online: http://www.nhc. gov.cn/yzygj/s7653p/202003/46c9294a7dfe4cef80dc7f5912 eb1989/files/ce3e6945832a438eaae415350a8ce964.pdf

25. Xu Y, Li X, Zhu B, et al. Characteristics of pediatric SARS$\mathrm{CoV}-2$ infection and potential evidence for persistent fecal viral shedding. Nat Med 2020;26:502-5.

26. Zeng L, Tao X, Yuan W, et al. First case of neonate infected with novel coronavirus pneumonia in China. Chin J Pediatr 2020. [Epub ahead of print].

Cite this article as: Xiao T, Cheng Y, Lu G, Zhou W. Pandemic considerations in pediatric critical care: what can we learn from COVID-19? Transl Pediatr 2021;10(10):2875-2880. doi: $10.21037 /$ tp-20-129
27. Castagnoli R, Votto M, Licari A, et al. Severe Acute Respiratory Syndrome Coronavirus 2 (SARS-CoV-2) Infection in Children and Adolescents: A Systematic Review. JAMA Pediatr 2020;174:882-9.

28. Zeng H, Xu C, Fan J, et al. Antibodies in Infants Born to Mothers With COVID-19 Pneumonia. JAMA 2020;323:1848-9.

29. Wölfel R, Corman VM, Guggemos W, et al. Virological assessment of hospitalized patients with COVID-2019. Nature 2020;581:465-9.

30. Shen K, Yang Y, Wang T, et al. Diagnosis, treatment, and prevention of 2019 novel coronavirus infection in children: experts' consensus statement. World J Pediatr 2020;16:223-31.

31. Xu Z, Shi L, Wang Y, et al. Pathological findings of COVID-19 associated with acute respiratory distress syndrome. Lancet Respir Med 2020;8:420-2.

32. Pediatric Acute Lung Injury Consensus Conference G. Pediatric acute respiratory distress syndrome: consensus recommendations from the Pediatric Acute Lung Injury Consensus Conference. Pediatr Crit Care Med 2015;16:428-39.

33. Sanders JM, Monogue ML, Jodlowski TZ, et al. Pharmacologic Treatments for Coronavirus Disease 2019 (COVID-19): A Review. JAMA 2020;323:1824-36.

34. Bashshur RL, Shannon GW, Bashshur N, et al. The Empirical Evidence for Telemedicine Interventions in Mental Disorders. Telemed J E Health 2016;22:87-113. 